https://doi.org/10.15407/ujpe63.10.934

C.M. SCHERBAKOV

Department for Theoretical Physics, Taras Shevchenko National University of Kyiv (2a, Glushkov Ave., Kyiv 03022, Ukraine; e-mail: kristian.for@gmail.com)

\title{
IMPACT OF THE FLEXOELECTRIC COUPLING AND ELECTROSTRICTION ON THE DISPERSION OF SOFT PHONON MODES AND NEUTRON SCATTERING IN FERROELECTRICS
}

\begin{abstract}
Within the Landau-Ginzburg-Devonshire (LGD) theory framework, the impact of the flexocoupling and electrostriction on the soft phonon dispersion and neutron scattering spectra is analyzed, and analytical expressions are derived. The impact of the higher gradient term in the LGD functional is studied analytically. The existence of incommensurate modulations in a temperature interval higher than the Curie temperature $T_{\mathrm{C}}$, but lower than the temperature of incommensurate phase transition $T_{\mathrm{IC}}, T_{\mathrm{C}}<T<T_{\mathrm{IC}}$, and under the condition of the flexocoefficient magnitude ranging over the critical value, $|f|>f^{\mathrm{cr}}(T)$, is established. The influence of the dynamic flexocoefficient $M$, suggested in [1], on phonon spectra is studied. We consider various parameters of the free energy functional; especially, we have discovered a significant contribution of the electrostriction to the appearance of a commensurate phase. In the recent years, the various methods based on the neutron scattering acquire more applications to study phonons in solid crystals. We have analyzed neutron scattering spectra and shown that theoretical predictions are in a very good agreement with observed data.
\end{abstract}

Ke ywords: flexocoupling, electrostriction, soft phonon dispersion, neutron scattering spectra, ferroelectrics.

\section{Introduction}

Physical properties of ferroics attract a permanent attention of researchers [2]. We would like to draw attention to the most striking interesting phenomena both for fundamental physics and applications induced by the flexoelectric effect. This effect predicted theoretically by Mashkevich and Tolpygo [3] in 1957 exists in any material, making the effect universal [1, 4-6]. Kvasov and Tagantsev [7] predicted the existence of a cross-term in the kinetic energy and named it the dynamic flexoelectric effect (see reviews $[1,5]$ and references therein).

The investigation of dynamic characteristics of phase transitions in ferroics [8] attracts a great attention of scientists for many years. Any phase transition leads to the instability of some phonon vibrations. In this case, the static displacements of atoms at the phase transition correspond to frozen displacements of the soft phonon vibration mode [9]. The frequency $\omega_{\text {TO }}$ of the transverse optic soft mode is temperature-

(c) C.M. SCHERBAKOV, 2018

934 dependent at the transition temperature $T=T_{\mathrm{c}}$. Experimental methods, which give information about the soft modes and the spatial modulation of the order parameter in ferroics are dielectric measurements [10], inelastic neutron scattering [9], [11-15], X-ray [16-18], Raman [19], and Brillouin [15, 16], [20-23] scatterings, and the pulse-echo method $[19,21]$.

The influence of static and dynamic flexoelectric couplings on the soft phonon spectra in ferroics has not been studied till the recent time [24-27], when the Landau-Ginzburg-Devonshire (LGD) approach was adopted to study the appearance of the spatially modulated phases (SMP) due to the flexocoupling and the properties of optic and acoustic phonons in the ferroelectric and paraelectric phases. Flexocoupling-induced soft acoustic modes and SMP in ferroelectrics were revealed in [26].

Within the LGD theory, we will analyze the expressions for the frequency dispersion of soft transverse acoustic (TA) and optic (TO) phonon modes in dependence on the flexoelectric coupling constant $f$ in ferroelectrics. We consider various parameters of

ISSN 2071-0194. Ukr. J. Phys. 2018. Vol. 63, No. 10 
the free energy functional; especially, we discover a significant contribution of the electrostriction to the appearance of a commensurate phase. In recent years, the various methods based on the neutron scattering acquire a more application to study phonons in solid crystals. Here, we will analyze the neutron scattering spectra and will show that theoretical predictions are in a very good agreement with observed data [26, 28].

\section{One-Component Approximation in the Free Energy Functional and the Lagrange Function}

The LGD free energy $F$ of a ferroic such as a proper or incipient ferroelectric acquires the simplest form for the one-component polarization, $\eta$, coupled with the strain tensor component, $u$, which depends only on the coordinate $x$ in the one-dimensional (1D) case [29]. In the simplest one-component 1D case considered hereinafter, the bulk part of the free energy $F$, which depends on $\eta, u$, and their gradients, has the form

$$
\begin{aligned}
& F_{V}=\int d x\left(\frac{\alpha(T)}{2} \eta^{2}+\frac{\beta}{4} \eta^{4}+\frac{\gamma}{6} \eta^{6}+\frac{g}{2}\left(\frac{\partial \eta}{\partial x}\right)^{2}+\right. \\
& +\frac{w}{2}\left(\frac{\partial^{2} \eta}{\partial x^{2}}\right)^{2}+\frac{h}{2} \eta^{2}\left(\frac{\partial \eta}{\partial x}\right)^{2}-\eta E-q u \eta^{2}+ \\
& \left.+\frac{c}{2} u^{2}+\frac{\nu}{2}\left(\frac{\partial u}{\partial x}\right)^{2}-\frac{f}{2}\left(\eta \frac{\partial u}{\partial x}-u \frac{\partial \eta}{\partial x}\right)\right) .
\end{aligned}
$$

The parameters $\alpha, \beta, \gamma, g, w, E, q, c, \nu, f$ were considered in [24-26]. On the contrary to those papers, we consider the non-zero gradient parameter $h$.

The Lagrange function $L=\int_{t} d t(F-K)$ includes the free energy $F$ given by Eq. (1) and the kinetic energy

$K=\int d x\left(\frac{\mu}{2}\left(\frac{\partial \eta}{\partial t}\right)^{2}+M \frac{\partial \eta}{\partial t} \frac{\partial U}{\partial t}+\frac{\rho}{2}\left(\frac{\partial U}{\partial t}\right)^{2}\right)$,

which involves the dynamic flexocoupling $[1,7]$ with the magnitude $M$. Here, $\rho$ is the density of a material, and $\mu$ is a kinetic coefficient. The elastic displacement component $U$ is connected with the strain $u$ through the relation $u=\frac{\partial U}{\partial x}$.

\section{Analytical Solutions of Linearized Equations of State}

The thermodynamic equations of state can be obtained from the variation of the free energy (1) in ISSN 2071-0194. Ukr. J. Phys. 2018. Vol. 63, No. 10 the components of the order parameter $\eta$ and strain $u, \frac{\delta F}{\delta \eta}=0$ and $\frac{\delta F}{\delta U}=0$. Let us find the solution of these equations after their linearization in vicinities of the spontaneous values $\eta_{s}$ and $u_{s}$

$\eta=\eta_{s}+\int d k \exp (i k x) \tilde{\eta}, \quad u=u_{s}+\int d k \exp (i k x) \tilde{u}$

The homogeneous spontaneous strain and order parameter values are denoted as $\eta_{s}$ and $u_{s}$, respectively. The perturbation field is $E=\int d k \exp (i k x) \tilde{E}$.

The spontaneous polarization and strain are absent $\left(\eta_{s}=0, u_{s}=0\right)$ in the high-temperature parent phase, wherein the coefficient $\alpha>0$. In the low-temperature ordered phase (wherein $\alpha<0$ and $\eta_{s} \neq 0, u_{s} \neq 0$ ), the spontaneous strain and polarization values can be determined from the equations of state, namely,

$u_{s}=\frac{q}{c} \eta_{s}^{2}, \quad \eta_{s}^{2}=\frac{1}{2 \gamma}\left(\sqrt{\beta^{* 2}-4 \alpha \gamma}-\beta^{*}\right)$,

where the coefficient

$\beta^{*}=\left(\beta-2 \frac{q^{2}}{c}\right)$.

Expression (4) is valid for ferroelectrics with the firstand second-order phase transitions from the ordered phase to the parent phase. In the particular case of the second-order phase transition for the parameters $\beta^{*}>0, \gamma=0$, the spontaneous polarization value is $\eta_{s}^{2}=-\alpha / \beta^{*}$, so the condition $c \beta>2 q^{2}$ should be valid for the transition realization.

In the particular case $\beta^{*}>0, \gamma=0$, the spontaneous value $\eta_{s}^{2}=\frac{-\alpha}{\beta^{*}}$. Therefore, the condition $c \beta>2 q^{2}$ should be valid for the second-order phase transition realization.

After the linearization, the equations of state acquire the form

$\tilde{\eta}=\tilde{\chi}(k) \tilde{E}, \quad \tilde{u}=-\frac{\left(i f k-2 q \eta_{s}\right)}{c+\nu k^{2}} \tilde{\chi}(k) \tilde{E}$.

The linear susceptibility $\tilde{\chi}$ introduced in Eq. (5) is given by the expression

$\tilde{\chi}(k)=\left(\alpha+3 \beta \eta_{s}^{2}+5 \gamma \eta_{s}^{4}-2 q u_{s}+\right.$
$\left.+g k^{2}+h k^{2} \eta_{s}^{2}+w k^{4}-\frac{4 q^{2} \eta_{s}^{2}+f^{2} k^{2}}{c+\nu k^{2}}\right)^{-1}$. 
The condition of instability of solution (5) corresponds to the divergence of susceptibility (6). By substituting expression (4) for $u_{s}$ into Eq. (6), we get the instability condition in the form

$\left(\alpha_{s}+g^{\mathrm{eff}} k^{2}+h k^{2} \eta_{s}^{2}+w k^{4}\right)\left(c+\nu k^{2}\right)-$ $-f^{2} k^{2}-4 q^{2} \eta_{s}^{2}=0$.

Here, we introduced the parameter $\alpha_{s}$ and effective gradient coefficient $g^{\text {eff }}$ :

$\alpha_{s}=\alpha+\left(3 \beta-2 \frac{q^{2}}{c}\right) \eta_{s}^{2}+5 \gamma \eta_{s}^{4}$

$g^{\mathrm{eff}}=g+h \eta_{s}^{2}+\frac{\alpha_{s} \nu}{c}-\frac{f^{2}}{c}$.

One can show that the parameter $\alpha_{s}$ is always positive. It is equal to $\alpha$ in the parent phase, wherein $\eta_{s}=0$. The inhomogeneous SMP can appear, if the renormalized gradient coefficient (9) becomes negative. Since the elastic stiffness is always positive $(c>$ $>0$ ), the homogeneous phase $\eta=\eta_{s}$ is absolutely stable under the condition $f^{2}<c g+c h \eta_{s}^{2}+\alpha_{s} \nu$. The condition is temperature-dependent because of the temperature dependence of the coefficient $\alpha_{s}(T)$.

If the strain gradient coefficient $\nu$ is zero, and the nonlinear gradient parameter $h$ is zero as well, the condition $f^{2}<c g+c h \eta_{s}^{2}+\alpha_{s} \nu$ reduces to the inequality $f^{2}<c g$, which is nothing more than the scalar form of the tensor relation $f_{k l m n}^{2}<g_{i j k l} c_{i j m n}$ from Ref. [30]. Later on, the condition $f_{44}<g_{44} c_{44}$ (along with other similar conditions) has been derived for the symmetry of perovskite by Yudin, Ahluwalia, and Tagantsev [31] and interpreted as the upper limit for the static flexoelectric tensor without the gradient $\frac{\nu_{i j k l m n}}{2}\left(\frac{\partial u_{i j}}{\partial x_{m}} \frac{\partial u_{k l}}{\partial x_{n}}\right)$ and other higher order gradients in the free energy of ferroelectrics. Mao and Purohit derived more complex stability conditions for the spatially confined systems with the strain gradient tensor $\nu_{i j k l m n} \neq 0$ [see Eq. (28) in Ref. [32]], which can be reduced to the simple inequality $f^{2}<\alpha \nu$. Stengel used the inequality $f^{2}<c g$ for the critical value of the flexoelectric coefficient [see Eq. (106) in Ref. [33]].

If one can neglect the smallest term $\nu w k^{6}$ in Eq. (7), it reduces to the biquadratic equation with four roots:

$\tilde{k}_{ \pm}=\left\{\frac{1}{2(1+\tilde{w})}\left(\tilde{f}^{2}-1-\tilde{\alpha}_{\nu} \pm\right.\right.$

$\left.\left.\pm\left\{\left(\tilde{f}^{2}-1-\tilde{\alpha}\right)^{2}-4 \tilde{\alpha}(1+\tilde{w})\left(1-\frac{\tilde{Q}^{2}}{\tilde{\alpha}}\right)\right\}^{1 / 2}\right)\right\}^{1 / 2}$ where we have introduced the dimensionless parameters $\tilde{k}, \tilde{f}, \tilde{\alpha}_{\nu}, \tilde{Q}, \tilde{w}$ :

$\tilde{k}=\sqrt{\frac{\nu}{c}} k, \quad \tilde{f}=\frac{f}{\sqrt{g_{s} c}}, \quad \tilde{\alpha}=\frac{\alpha_{s} \nu}{g_{s} c}$,

$\tilde{Q}=\frac{2 \eta_{s}}{c} \sqrt{\frac{\nu}{g_{s}}} q, \quad \tilde{w}=\frac{c w}{\nu g_{s}}$,

and $g_{s}$ is renormalized parameter defined as $g_{s}=g+$ $+h \eta_{s}^{2}$. Subscripts 1 and 2 in expression (10) correspond to the "+" and "-" signs before the outer square root. The solution $k_{1,2}^{+}$corresponds to the "+ + sign before the inner square root, and the root $k_{1,2}^{-}$corresponds to the "-" sign before it.

Expression (9) for $g^{\text {eff }}$ written in the dimensionless variables (11) acquires the form $g^{\text {eff }}=g_{s}(1+$ $\left.+\tilde{\alpha}-\tilde{f}^{2}\right)$. Thus, the necessary condition of the SMP appearance $g^{\text {eff }}<0$, written in the dimensionless variables (11), is equivalent to the inequality $\operatorname{sign}\left(g_{s}\right)\left(1+\tilde{\alpha}-\tilde{f}^{2}\right)<0$. However, as one can see from Eq. (10), the condition $g^{\text {eff }}<0$ is not sufficient per se, because the additional condition of the positive inner determinant,

$\left(\tilde{f}^{2}-1-\tilde{\alpha}\right)^{2} \geq 4 \tilde{\alpha}(1+\tilde{w})\left(1-\frac{\tilde{Q}^{2}}{\tilde{\alpha}}\right)$,

should be valid. Taking into account that the gradient parameter $h$ can accept positive, as well as negative, values, we note that the parameter $g_{s}$ which has the form $g_{s}=g+h \eta_{s}^{2}$ for the given parameter $g$ can effectively be overriden and switch it sign to the opposite value, so one can additionaly control the stability of SMP by the nonlinear gradient parameter $h$.

\section{Soft Phonon Dispersion in Ferroics with CP and ICP}

Soft phonon dispersion can be calculated from the time-dependent dynamic equations of state for the polarization and the elastic displacement components $\eta$ and $U$, respectively [24]. The nonlinear gradient parameter $w$ is usually small, so its influence will be neglected hereinafter. The dynamic equations of state are obtained from the variation of the Lagrange function $L=\int_{t} d t(F-K)$ in $\eta$ and $U$, where the free energy $F$ is given by Eq. (1), and the kinetic energy $K$ is given by Eq. (2). The solutions of the dynamic equations $\frac{\delta L}{\delta U}=0$ and $\frac{\delta L}{\delta \eta}=0$ can be found in the spatial $(k)$ and frequency $(\omega)$ domains, using Fourier

ISSN 2071-0194. Ukr. J. Phys. 2018. Vol. 63, No. 10 
integrals for the polarization, $\eta=\eta_{s}+\int d k \exp (i k x+$ $+i \omega t) \tilde{\eta}$, displacement, $U=u_{s} x+\int d k \exp (i k x+$ $+i \omega t) \tilde{U}$, and perturbation field, $E=\int d k \exp (i k x+$ $+i \omega t) \tilde{E}$ after the linearization in vicinities of the spontaneous values $\eta_{s}$ and $u_{s}$, respectively.

The generalized linear susceptibility function $\tilde{\chi}(k, \omega)$ is defined by the expression $\tilde{\eta}=\tilde{\chi}(k, \omega) \tilde{E}$. The condition of the susceptibility divergence leads to the equation for the phonon dispersion $\omega(k)$, which can be defined by a power series of the form

$\tilde{k}^{6}+\left(\tilde{\alpha}+1-\tilde{f}^{2}-\tilde{\mu} \tilde{\omega}^{2}\right) \tilde{k}^{4}+\left(\tilde{\alpha}-\tilde{Q}^{2}-\right.$

$\left.-(1-2 \tilde{M} \tilde{f}+\tilde{\mu}) \tilde{\omega}^{2}\right) \tilde{k}^{2}-\left(\tilde{\alpha}-\left(\tilde{\mu}-\tilde{M}^{2}\right) \tilde{\omega}^{2}\right) \tilde{\omega}^{2}=0$

where the parameters $\tilde{k}, \tilde{f}, \tilde{\alpha}$, and $\tilde{Q}$ were initially defined in Eq. (11). We have introduced here the dimensionless frequency $\tilde{\omega}$ and the parameters $\tilde{M}$ and $\tilde{\mu}$ in the following way:

$\tilde{\omega}=\frac{\sqrt{\rho \nu}}{c} \omega, \quad \tilde{M}=\frac{M}{\rho} \sqrt{\frac{c}{g_{s}}}, \quad \tilde{\mu}=\frac{\mu c}{\rho g_{s}}$.

In what follows, we are going to consider the bicubic equation (12) for the wave vector, rather than a biquadratic one for the frequency. There are a few important reasons for why we have decided to switch to studying the dependence of the wave vector on the frequency, but not vice versa.

First of all, on the contrary to the biquadratic equation case, we receive yet another solution here, a quite different branch of oscillations. As can be seen from our previous papers [24-27], the biquadratic equation for a frequency give us two well-known solutions: the acoustic branch with linear dispersion relation in vicinities of small wave vectors and the optic branch with non-zero oscillations at the zero wave number $k=0$. From the bicubic equation (12), we get the third independent solution, which describes the relaxation oscillations. This means that there is a damping in the system that can be measured and studied by experimental observations. It is impossible just to neglect or ignore this solution, and it is mandatory to consider the relaxations in the system to complete our full analysis. So far, solutions of the bicubic equation (12) for the wave vector give us a full analytical description of the system behavior.

The second important reason for why we prefer to study the bicubic equation for the wave vector is experimental observations of the neutron scattering on samples of the considering systems, which give us the dependence of the intensity of scattered ray beams on the frequency. So far, in order to compare experimental observations with the our theoretical predictions, we should find out the dependence of the wave number on the wave frequency.

\section{Neutron Scattering}

The structure factors are the numbers that express the amplitude of reflection from the surface. The total scattering power of all atoms is given by the sum of the individual scattering amplitudes [see Eq. (8.2.43) in Ref. [34]]: $S_{k}=\frac{1}{N}\left|\sum_{j=1}^{N} e^{i k r_{j}}\right|^{2}$, where we have marked the $j$ th atom by index $j$, and $N$ is the total number of atoms in the system. The intensity of reflection from the surface has the direct relation to the structure factors: $I \sim S_{k}$ [see Eq. (8.2.46) in Ref. [34]].

In the harmonic approximation, the intensity of the scattered ray beams has the form (see Appendix A)

$I \sim \frac{k^{2}}{\omega^{2}}$

In order to consider the behavior of the dispersion relation in the region of small wave vectors, we can neglect the term $\sim \tilde{k}^{6}$ in Eq. (12), so the dispersion relation has the form

$\tilde{k}^{2}=-\frac{\tilde{\lambda}_{\omega}}{2 \tilde{\delta}_{\omega}} \pm \frac{\sqrt{\tilde{\lambda}_{\omega}^{2}+4 \tilde{\delta}_{\omega}\left(\tilde{\alpha}-\left(\tilde{\mu}-\tilde{M}^{2}\right) \tilde{\omega}^{2}\right)}}{2 \tilde{\delta}_{\omega}}$,

where $\tilde{\lambda}_{\omega}=\tilde{\alpha}-\tilde{Q}^{2}-(1-2 \tilde{M} \tilde{f}+\tilde{\mu}) \tilde{\omega}^{2}, \tilde{\delta}_{\omega}=(\tilde{\alpha}+1-$ $\left.-\tilde{f}^{2}-\tilde{\mu} \tilde{\omega}^{2}\right)$. As it will be shown below, the solution with "+" before the square root corresponds to the acoustic dispersion and that with "-." corresponds to the optic one (see explanations to Eq. (21)).

Experimental data on the neutron scattering intensity are presented in Fig. 1. There was raised the question about the nature of the increase near $T_{\mathrm{IC}}$ in the Ref. [29] (see Fig. 1). It was suggested that the second-order phase transition becomes a first-order one, which relates to the strain coupling [35]. It will be shown in Section 8 that the electrostriction itself may be responsible for the intensity pick widening.

\section{Acoustic Dispersion}

Ar small frequencies and small wave vectors, the dispersion relation (12) acquires the linear form

$\omega=\sqrt{\frac{c}{\rho}\left(1-4 \frac{\eta_{s}^{2} q^{2}}{c \alpha_{s}}\right)} k$, 

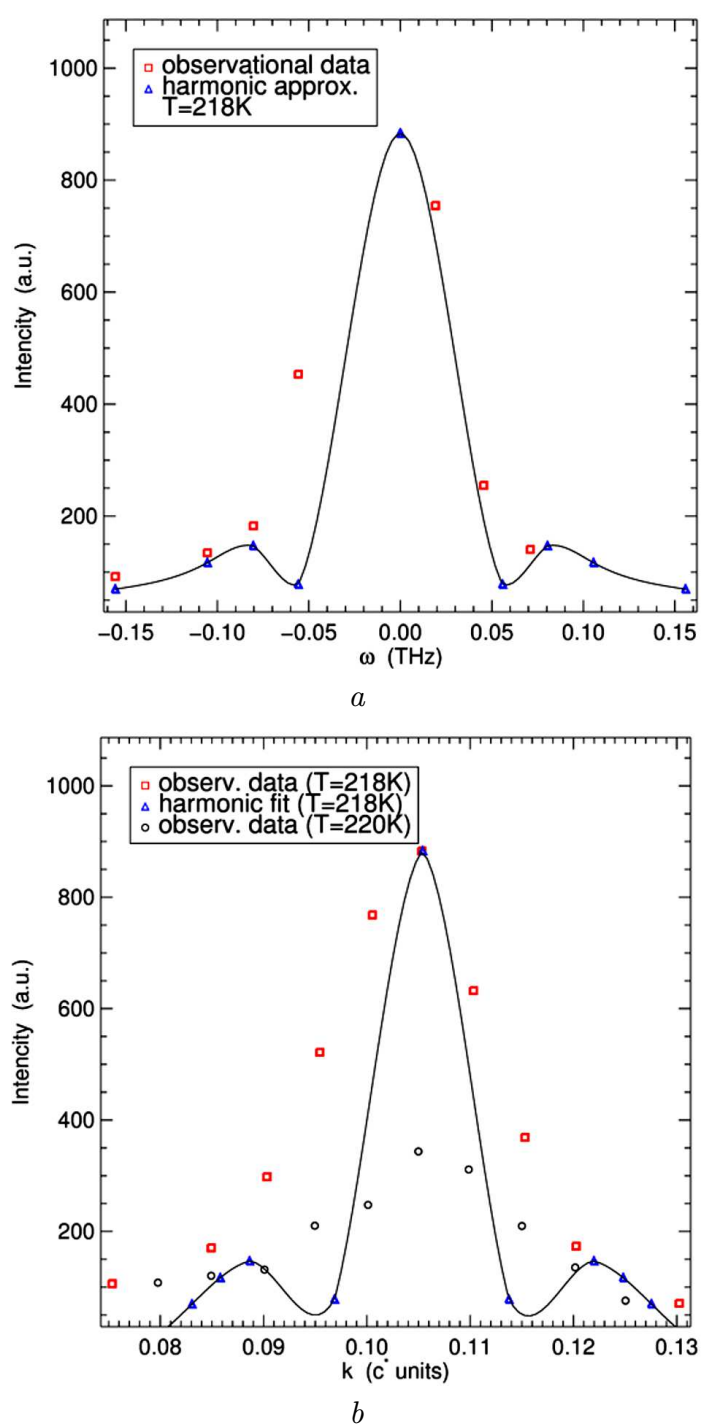

Fig. 1. Neutron scattering intensity observational data and predicted values in the harmonic approximation. Experimental data are denoted by red squares, and theoretical predictions are given as blue triangles with eye-guiding solid line. Experimental data are taken from Ref. [29], our modeling is based on Eq. (14). Intensity is given in arbitrary units (a.u.). It sharply increases, by approaching the phase transition $(a)$. The satellite Bragg reflection shows the additional intensity pick below $T_{\text {IC }}(b)$

which represents the acoustic dispersion law. Combining this result with Eq. (14), we can find out the most important thing about the neutron scattering, namely the temperature resonance of the intensity of scattered rays when the temperature constant $\alpha_{s}$ ap- proaches the squared electrostriction: $\alpha_{s}=4 \frac{\eta_{s}^{2}}{c} q^{2}$ $\left(\tilde{\alpha}=\tilde{Q}^{2}\right)$. This transition takes place at the temperature $T_{\mathrm{IC}}$ of the transition from the commensurate phase to an incommensurate one and can be found by equating the r.h.s. of expression (16) to zero. In view of Eq. (4), we have

$T_{\mathrm{IC}}=T_{C}+\frac{\left(\beta-2 \frac{q^{2}}{c}\right)^{2}}{4 \gamma \alpha_{T}}$.

According to Eq. (16) and Eq. (17), the softening of the acoustic mode occurs at the temperature $T_{\mathrm{IC}}$ that is always higher than the Curie temperature $T_{\mathrm{C}}$ : $T_{\mathrm{IC}}>T_{\mathrm{C}}$.

There is also the second solution $T_{\mathrm{IC}}=T_{\mathrm{C}}$. This kind of ferroics undergoes a direct secondorder phase transition at the Curie point $T_{\mathrm{C}}$. The $\mathrm{Sn}_{2} \mathrm{P}_{2}\left(\mathrm{~S}_{1-x} \mathrm{Se}_{x}\right)_{6}$ family of uniaxial ferroelectrics is an excellent model system for studying the appearance of an intermediate IC phase, since the mixed crystals can be grown for all values of $x(0<$ $<x<1) . \mathrm{Sn}_{2} \mathrm{P}_{2} \mathrm{Se}_{6}$ shows an intermediate incommensurate phase at ambient pressure, in contrast to $\mathrm{Sn}_{2} \mathrm{P}_{2} \mathrm{~S}_{6}[29]$.

Equation (17) allows us to plot the dependence of an incommensurate phase transition temperature $T_{\text {IC }}$ vs the electrostriction. There is a slight dependence in the region of small electrostriction values. But, as the electrostriction parameter increases, one can notice a strong increase of the phase transition temperature $T_{\mathrm{IC}}$. This temperature was reported in [29] for a sample of $\mathrm{Sn}_{2} \mathrm{P}_{2} \mathrm{Se}_{6}: T_{\mathrm{IC}}=221 \mathrm{~K}$. Therefore, we can find the electrostriction $q=6 \times 10^{9} \mathrm{Jm} / \mathrm{C}^{2}$ in this case. One can retrieve it directly from Eq. (17) by solving a simple quadratic equation as well. Note that, at the point $q \approx 0$, the temperature $T_{\mathrm{IC}}$ equals $193.26 \mathrm{~K}$. It is just $0.26 \mathrm{~K}$ above the Curie point.

The plots of the phase transition temperature $T_{\mathrm{IC}}$ (lower panel) and the sound velocity dependence on the electrostriction (upper panel) are presented in Fig. 2.

\section{Phonon Damping}

In order to find out the condition of the appearance of a damping in a system, one should examine the damping region by the standard replacement $\omega \rightarrow i \omega$ in the Eq. (15). Accordingly, the critical frequency of the appearance of the damping in a system can be 
found by setting the expression under the square root in Eq. (15) equal to zero

$$
\begin{aligned}
& \tilde{\omega}_{\mathrm{damp}}^{\mathrm{cr}}= \\
& =\frac{\tilde{\alpha}-\tilde{Q}^{2}}{\sqrt{4\left(\tilde{\alpha}-\tilde{f}^{2}+1\right) \tilde{\alpha}-2(1+\tilde{\mu}-2 \tilde{M} \tilde{f})\left(\tilde{\alpha}-\tilde{Q}^{2}\right)}} .
\end{aligned}
$$

It is possible to suggest that the appearance of the damping in a system has the direct influence on the intensity of scattered rays and leads to a softening of the acoustic mode. It causes the appearance of the main pick of the structure factor. Taking into account that the central peak increases in intensity, by approaching the phase transition, and shows a slight narrowing, as $\omega_{\text {damp }}^{\text {cr }}$ does, it is possible that the line width of the structure factor is close to $\omega_{\text {damp }}^{\text {cr }}$, which decreases, when the temperature $T$ approaches the phase transition temperature $T_{\mathrm{IC}}$.

\section{Soft Optic Mode}

Near the phase transition temperature $T_{\mathrm{IC}}\left(\tilde{\alpha} \approx \tilde{Q}^{2}\right)$, the dispersion relation at small wave vectors and nonzero frequencies in accordance with Eq. (12) has the form:

$\left(\tilde{\mu}-\tilde{M}^{2}\right) \tilde{\omega}^{2}=\tilde{\alpha}+(1-2 \tilde{M} \tilde{f}+\tilde{\mu}) \tilde{k}^{2}$.

So we have the Lorentz type of Green's function. The case where the wave vector approaches zero is of special interest, because this allows us to determine the frequency at zero point. So far, we have an important fitting expression

$\tilde{\omega}_{o}=\sqrt{\frac{\tilde{\alpha}}{\tilde{\mu}-\tilde{M}^{2}}}$,

where $\tilde{\omega}_{o}=\tilde{\omega}_{k=0}$. The parameter $\omega_{o}$ can be determined as the frequency at $k=0$. The dispersion relation has singularity at the point of the critical dynamic flexoconstant $M^{\mathrm{cr}}=\sqrt{\rho \mu}$. In accordance with Eq. (19), the square of the frequency linearly depends on the square of the wave vector: $\omega^{2}=\omega_{k=0}^{2}+c_{o}^{2} k^{2}$, where the upper limit of the phonon velocity $c_{o}$ can be expressed as $c_{o}=\tilde{c}_{o} \sqrt{\frac{c}{\rho}}$. The dimensionless speed variable $\tilde{c}_{o}$ has the form

$\tilde{c}_{o}=\sqrt{\frac{1-2 \tilde{M} \tilde{f}+\tilde{\mu}}{\tilde{\mu}-\tilde{M}^{2}}}$.

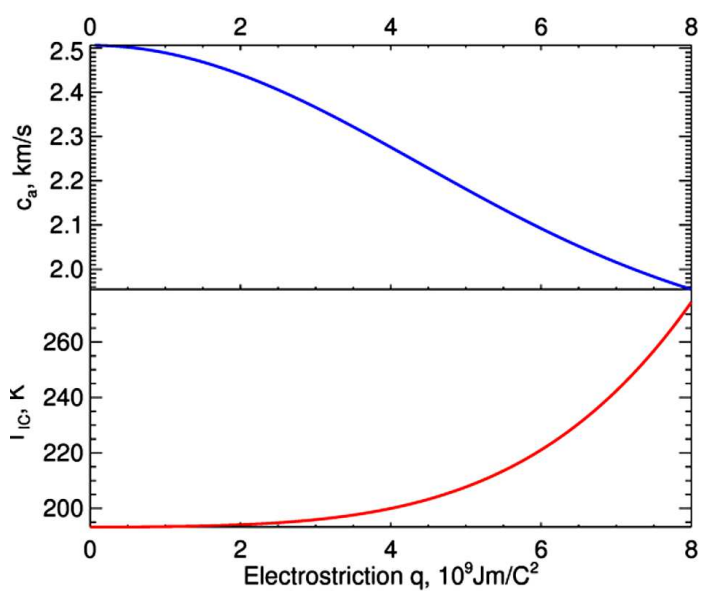

Fig. 2. Influence of the electrostriction on the sound speed $c_{a}$ (upper panel) and incommensurate phase transition temperature $T_{\mathrm{IC}}$ (lower panel). Numerical calculations were performed for a $\mathrm{Sn}_{2} \mathrm{P}_{2} \mathrm{Se}_{6}$ sample. The parameters $\beta, c, T_{\mathrm{C}}, \alpha_{T}, \gamma, \rho$ are listed in the Table. The sound velocity as a function of the electrostriction was calculated at a temperature $T=100 \mathrm{~K}$

The parameter $c_{o}^{2}$ can be determined as the tangent of an angle of the dispersion curve in the range of small values of the wave vector on the plane $\omega^{2}-k^{2}$.

The expression $(1-2 \tilde{M} \tilde{f}+\tilde{\mu})$ under the square root in Eq. (21) should be positive definite. So, the quantity $\tilde{\lambda}_{\omega}$ in Eq. (15) has a negative value, and we can conclude that the sign "-" before the square root in Eq. (15) defines the optic branch, as well as sign "+" before the square root determines the acoustic dispersion.

Note that, for the large enough wave vectors and frequencies, the term $\tilde{\alpha}$ can be neglected in Eq. (19). In this particular case, we have the linear phonon dispersion relation $\omega=c_{o} k$.

One can determine previously unknown parameters of the kinetic coefficient $\tilde{\mu}$ and the dynamic flexocoefficient $\tilde{M}$ by combining Eqs. (20) and (21):

$$
\begin{aligned}
& \tilde{M}^{ \pm}=\tilde{f} \pm \sqrt{\tilde{f}^{2}-1+\frac{\left(-1+{\tilde{c_{o}}}^{2}\right) \tilde{\alpha}}{{\tilde{\omega_{o}}}^{2}}}, \\
& \tilde{\mu}^{ \pm}=\left(\tilde{f} \pm \sqrt{\tilde{f}^{2}-1+\frac{\left(-1+{\tilde{c_{o}}}^{2}\right) \tilde{\alpha}}{\tilde{\omega}_{o}^{2}}}\right)^{2}+\frac{\tilde{\alpha}}{\tilde{\omega}_{o}^{2}} .
\end{aligned}
$$

These equations unveil a degenerate phonon energy level with a frequency $\tilde{\omega}_{o}$ at $k=0$. It corresponds to the different sets of the parameters $\left\{\mu^{+}, M^{+}\right\}$and $\left\{\mu^{-}, M^{-}\right\}$. Such a finding can point out to a possi- 
Model parameters in the LGD free energy (1) and the kinetic energy (2) for several ferroelectrics with $g>0$ and $g<0$

\begin{tabular}{|c|c|c|}
\hline Symbol and dimension & $\mathrm{Sn}_{2} \mathrm{P}_{2} \mathrm{~S}_{6}$ & $\mathrm{Sn}_{2} \mathrm{P}_{2} \mathrm{Se}_{6}$ \\
\hline$\alpha_{T}\left(\times 10^{5} \mathrm{Jm} / \mathrm{Kc}^{2}\right)$ & 16 & 26 \\
\hline$T_{\mathrm{C}}(\mathrm{K})$ & 337 & 193 \\
\hline$T_{\mathrm{IC}}(\mathrm{K})$ & - & 221 \\
\hline$\beta\left(\times 10^{8} \mathrm{JC}^{-4} \mathrm{~m}^{5}\right)$ & +7.42 & -4.8 \\
\hline$\gamma\left(\times 10^{9} \mathrm{JC}^{-6} \mathrm{~m}^{9}\right)$ & 35 & 85 \\
\hline$q\left(\times 10^{9} \mathrm{Jm} / \mathrm{C}^{2}\right)$ & 4 (reference interval 1.6-4.7) & 6 \\
\hline$c\left(\times 10^{10} \mathrm{~Pa}\right)$ & $1.6\left(\right.$ since $\left.c_{44}=1.6 \pm 0.3\right)$ & 1.6 \\
\hline$g\left(\times 10^{-10} \mathrm{C}^{-2} \mathrm{~m}^{3} \mathrm{~J}\right)$ & 0.5 (fitting parameter) & -0.6 (fitting parameter) \\
\hline$w\left(\times 10^{-29} \mathrm{Jm}^{5} / \mathrm{C}^{2}\right)$ & 1.8 (reference value 1.8 ) & 2.5 (reference value 2.2 ) \\
\hline$\nu\left(\times 10^{-9} \mathrm{Vs}^{2} / \mathrm{m}^{2}\right)$ & 5 (fitting parameter) & 1 (fitting parameter) \\
\hline$f(\mathrm{~V})$ & $\pm(1.6-1.8)$ (fitting parameter) & \pm 1.0 (fitting parameter) \\
\hline$\rho\left(\times 10^{3} \mathrm{~kg} / \mathrm{m}^{3}\right)$ & 1.801 & 2.547 \\
\hline$\mu\left(\times 10^{-18} \mathrm{~s}^{2} \mathrm{~mJ}\right)$ & 11.0 (fitting parameter) & 14.5 (fitting parameter) \\
\hline$M\left(\times 10^{-8} \mathrm{Vs}^{2} / \mathrm{m}^{2}\right)$ & \pm 2.5 (fitting parameter) & \pm 1.5 (fitting parameter) \\
\hline
\end{tabular}

ble phonon energy level splitting in the strong external fields. From Eqs. (22) and (23), one can conclude that the nonzero dynamic flexocoefficient $M$ causes degenerate phonon spectra, and a further research is required to provide evidence of the influence of the dynamic flexoelectric effect on the dispersion relation.

\section{Summary}

We have studied the contribution made by the higher gradient term $h$ in the LGD functional to the appearance of SMP. We have analyzed the dynamic flexocoefficient $M$ and proposed a way how its value can be extracted from the phonon spectra. We have discovered how the critical temperature $T_{\mathrm{IC}}$ depends on various parameters of the free energy functional, with a special emphasis on the electrostriction, which significantly contributes to the appearance of a commensurate phase. In the recent years, various methods based on the neutron scattering acquire more applications to study phonons in solid crystals. Here, we have analyzed the neutron scattering spectra and shown that theoretical predictions are in a very good agreement with observed data.

\section{APPENDIX A.}

\section{Structure Factor}

The structure factors are the numbers that express the amplitude of reflection from the surface. The total scattering power of all atoms is given by the sum of the individual scattering amplitudes [see Eq. (8.2.43) in Ref. [34]]: $S_{k}=\frac{1}{N}\left|\sum_{j=1}^{N} e^{i k r_{j}}\right|^{2}$, where we have denoted the $j$ th atom by index $j$, and $N$ is the total number of atoms in the system. The intensity of reflection from the surface has the direct relation to the structure factors: $I \sim S_{k}$ [see Eq. (8.2.46) in Ref. [34]].

Let us introduce collective variables $\rho_{k}: \rho_{k}=\frac{1}{\sqrt{N}} \times$ $\times \sum_{j=1}^{N} e^{-i k r_{j}}$. Note that $S_{k}=\left|\rho_{k}\right|^{2}$.

In the harmonic approximation,

$\left|\dot{\rho}_{k}\right|^{2}=\omega^{2}\left|\rho_{k}\right|^{2}$.

On the other hand, the direct derivation gives us the expression

$\left|\dot{\rho}_{k}\right|^{2}=\frac{1}{N} \sum_{j=1}^{N}\left(k \dot{r}_{j}\right)^{2}+\frac{1}{N} \sum_{i \neq j} \sum_{k}\left(k \dot{r}_{i}\right)\left(k \dot{r}_{j}\right) e^{-i k\left(r_{i}-r_{j}\right)}$.

Averaging r.h.s. over speeds, we finally find the expression for structure factors: $\omega^{2} S_{k}=k^{2}\left\langle v^{2}\right\rangle$, where $\left\langle v^{2}\right\rangle$ is the averaged squared speed, and we have obtained, in a natural way, the intensity of scattered neutron ray beams:

$I \sim \frac{k^{2}}{\omega^{2}}$.

1. P.V. Yudin, A.K. Tagantsev. Fundamentals of exoelectricity in solids. Nanotechnology 24, 432001 (2013).

2. M.D. Glinchuk, A.V. Ragulya, V.A. Stephanovich. Nanoferroics (Springer, 2013), p. 378.

3. V.S. Mashkevich, K.B. Tolpygo. The interaction of vibrations of nonpolar crystals with electric fields. Sov. Phys. JETP 4, 455 (1957).

ISSN 2071-0194. Ukr. J. Phys. 2018. Vol. 63, No. 10 
4. A.K. Tagantsev. Piezoelectricity and exoelectricity in crystalline dielectrics. Phys. Rev. B 34, 5883 (1986).

5. P. Zubko, G. Catalan, A.K. Tagantsev. Flexoelectric effect in solids. Ann. Rev. Mater. Research 43, 387 (2013).

6. S.V. Kalinin, A.N. Morozovska. Multiferroics: Focusing the light on exoelectricity (comment). Nature Nanotechnology 10, 916 (2015).

7. A. Kvasov, A.K. Tagantsev. Dynamic exoelectric effect in perovskites from first-principles calculations. Phys. Rev. B 92, 054104 (2015).

8. W. Cochran. Crystal stability and the theory of ferroelectricity. Phys. Rev. Lett. 3, 412 (1959).

9. G. Shirane, J.D. Axe, J. Harada, J.P. Remeika. Soft ferroelectric modes in lead titanate. Phys. Rev. B 2, 155 (1970).

10. W. Cochran. Dynamical scattering and dielectric properties of ferroelectric crystals. Adv. in Phys. 18, 157 (1969).

11. G. Shirane, Y. Yamada. Lattice-dynamical study of the $110 \mathrm{~K}$ phase transition in $\mathrm{SrTiO}_{3}$. Phys. Rev. 177, 858 (1969).

12. R. Currat, H. Buhay, C.H. Perry, A.M. Quittet. Inelastic neutron scattering study of anharmonic interactions in orthorhombic $\mathrm{KNbO}_{3}$. Phys. Rev. B 40, 10741 (1989).

13. I. Etxebarria, M. Quilichini, J.M. Perez-Mato, P. Boutrouille, F.J. Zuniga, T. Breczewski. Inelastic neutron scattering investigation of external modes in incommensurate and commensurate $\mathrm{A}_{2} \mathrm{BX}_{4}$ materials. J. Phys.: Condens. Matter 4, 8551 (1992).

14. J. Hlinka, M. Quilichini, R. Currat, J.F. Legrand, Dynamical properties of the normal phase of betaine calcium chloride dihydrate. I. Experimental results. J. Phys.: Condens. Matter 8, 8207 (1996).

15. J. Hlinka, S. Kamba, J. Petzelt, J. Kulda, C.A. Randall, S.J. Zhang. Origin of the "waterfall" effect in phonon dispersion of relaxor perovskites. Phys. Rev. Lett. 91, 107602 (2003).

16. V. Goian, S. Kamba, O. Pacherova, J. Drahokoupil, L. Palatinus, M. Dušek, J. Rohlček, M. Savinov, F. Laufek, W. Schranz, A. Fuith, M. Kachlk, K. Maca, A. Shkabko, L. Sagarna, A. Weidenkaff, A.A. Belik. Antiferrodistortive phase transition in $\mathrm{EuTiO}_{3}$. Phys. Rev. B 86, 054112 (2012).

17. Jong-Woo Kim, P. Thompson, S. Brown, P.S. Normile, J.A. Schlueter, A. Shkabko, A. Weidenkaff, P.J. Ryan. Emergent superstructural dynamic order due to competing antiferroelectric and antiferrodistortive instabilities in bulk $\mathrm{EuTiO}_{3}$. Phys. Rev. Lett. 110, 027201 (2013).

18. R.G. Burkovsky, A.K. Tagantsev, K. Vaideeswaran, N. Setter, S.B. Vakhrushev, A.V. Filimonov, A. Shaganov et al. Lattice dynamics and antiferroelectricity in $\mathrm{PbZrO}_{3}$ tested by x-ray and Brillouin light scattering. Phys. Rev. B 90, 144301 (2014).

19. J. Hlinka, I. Gregora, V. Vorlcek. Complete spectrum of long-wavelength phonon modes in $\mathrm{Sn}_{2} \mathrm{P}_{2} \mathrm{~S}_{6}$ by Raman scattering. Phys. Rev. B 65, 064308 (2002).

20. A. Kohutych, R. Yevych, S. Perechinskii, V. Samulionis, J. Banys, Yu. Vysochanskii. Sound behavior near the
Lifshitz point in proper ferroelectrics. Phys. Rev. B 82, 054101 (2010).

21. A. Kohutych, R. Yevych, S. Perechinskii, Y. Vysochanskii. Acoustic attenuation in ferroelectric $\mathrm{Sn}_{2} \mathrm{P}_{2} \mathrm{~S}_{6}$ crystals. Open Physics 8, 905 (2010).

22. Yu.M. Vysochanskii, A.A. Kohutych, A.V. Kityk, A.V. Zadorozhna, M.M. Khoma, A.A. Grabar. Tricritical behavior of $\mathrm{Sn}_{2} \mathrm{P}_{2} \mathrm{~S}_{6}$ ferroelectrics at hydrostatic pressure. Ferroelectrics 399, 83 (2010).

23. R.M. Yevych, Yu.M. Vysochanskii, M.M. Khoma, S.I. Perechinskii. Lattice instability at phase transitions near the Lifshitz point in proper monoclinic ferroelectrics. J. Phys.: Condens. Matter 18, 4047 (2006).

24. A.N. Morozovska, Yu.M. Vysochanskii, O.V. Varenyk, M.V. Silibin, S.V. Kalinin, E.A. Eliseev. Flexocoupling impact on the generalized susceptibility and soft phonon modes in the ordered phase of ferroics. Phys. Rev. B 92, 094308 (2015).

25. A.N. Morozovska, E.A. Eliseev, C.M. Scherbakov, Yu.M. Vysochanskii. The influence of elastic strain gradient on the upper limit of exocoupling strength, spatiallymodulated phases and soft phonon dispersion in ferroics. Phys. Rev. B 94, 174112 (2016).

26. A.N. Morozovska, M.D. Glinchuk, E.A. Eliseev, Yu.M. Vysochanskii. Flexocoupling-induced soft acoustic mode and the spatially modulated phases in ferroelectrics. Phys. Rev. B 96, 094111 (2017).

27. A.N. Morozovska, V.V. Khist, M.D. Glinchuk, C.M. Scherbakov, M.V. Silibin, D.V. Karpinsky, E.A. Eliseev. Flexoelectricity-induced spatially modulated phases in ferroics and liquid crystals. J. Molecul. Liq. 267, 550 (2018); arXiv:1710.01033.

28. L.D. Landau, E.M. Lifshitz. Theory of Elasticity. Theoretical Physics (Butterworth-Heinemann, 1998), Vol. 7.

29. S.W.H. Eijt, R. Currat, J.E. Lorenzo, P. Saint-Gregoire, B. Hennion, Yu.M. Vysochanskii. Soft modes and phonon interactions in Sn2P2S6 studied by neutron scattering. Eur. Phys. J. B 5, 169 (1998).

30. E.A. Eliseev, A.N. Morozovska, M.D. Glinchuk, R. Blinc. Spontaneous exoelectric/exomagnetic effect in nanoferroics. Phys. Rev. B 79, 165433 (2009).

31. P.V. Yudin, R. Ahluwalia, A.K. Tagantsev. Upper bounds for exocoupling coeffcients in ferroelectrics. Appl. Phys. Lett. 104, 082913 (2014).

32. S. Mao, P.K. Purohit. Insights into exoelectric solids from strain-gradient elasticity. J. Appl. Mech. 81, 081004 (2014).

33. M. Stengel. Unified ab initio formulation of exoelectricity and strain-gradient elasticity. Phys. Rev. B 93, 245107 (2016).

34. N.H. March, W.H. Young, S. Sampanthar. The Many-Body Problem in Quantum Mechanics (Cambridge Univ. Press, 1967).

35. C.P. Bean, D.S. Rodbell. Magnetic disorder as a first-order phase transformation. Phys. Rev. B 126, 104 (1962).

Received 10.06.18 


\section{К. Щербаков}

\section{ВПЛИВ ФЛЕКСОЗВ'ЯЗКУ}

ТА ЕЛЕКТРОСТРИКЦІЇ НА ДИСПЕРСІЮ

М'ЯКИХ ФОНОННИХ МОД ТА РОЗСІЯННЯ НЕЙТРОНІВ В СЕГНЕТОЕЛЕКТРИКАХ

$\mathrm{P}$ е 3 ю м е

В рамках формалізму Ландау-Гінзбурга-Девоншира (ЛГД) було встановлено та проаналізовано вирази, що описують дисперсію м'яких фононів, спектри розсіяння нейтронів в залежності від флексозв'язку та електрострикції. Також аналітично вивчено вплив вищих градієнтних доданків в ЛГД функціоналі. Встановлено існування неспівмірних модуляцій у температурному діапазоні вище темпе- ратури Кюрі $T_{\mathrm{C}}$, але нижче за температуру переходу в неспівмірну фазу $T_{\mathrm{IC}}, T_{\mathrm{C}}<T<T_{\mathrm{IC}}$, та при умові, що абсолютна величина флексокоефіцієнта $f \in$ вищою за деяке критичне значення $f^{\mathrm{cr}}(T),|f|>f^{\mathrm{cr}}(T)$. Вивчено вплив динамічного флексокоефіцієнта $M$, запропонованого в [1], на фононні спектри. В роботі ми аналізуємо різні параметри ЛГД функціонала i, що є суттєвим, показали що електрострикція дає суттєвий внесок в появу співмірної фази. В останні роки спостерігається значний розвиток різних методів, що грунтуються на технологіях розсіювання нейтронів і потребують ширшого вивчення фононів в твердих тілах. В статті було проаналізовано експериментальні дані розсіювання нейтронів і показано, що теоретичні розрахунки добре узгоджені із спостережуваними даними. 